\title{
Work Rates of International Hockey Umpires
}

Christopher A Spice, Dan A Gordon, Lee Smith, James A Johnstone.

Cambridge Centrefor Sport and Exercise Sciences, Anglia Ruskin University

Corresponding Author

Christopher A Spice,

Anglia Ruskin University, East Road, Cambridge, CB1 1PT,

08451962124,

chris.spice@anglia.ac.uk 


\section{ORIGINAL ARTICLE}

Work Rates of International Hockey Umpires 


\section{Abstract}

In recent years, a number of significant changes have occurred to the rules of international hockey, but no evaluation has taken place of the effect this has had on the demands placed upon match officials. The present study assessed match demands placed on elite male and female hockey officials. A total of 10 male and 11 female officials were 'tracked' using Global Positioning System receivers for 66 international matches. Data extracted from receivers included metrics for distances covered during the game and while engaged in high intensity running (HIR), as well as characteristics of each individual effort. Differences between sex and time periods were assessed using Kruskal-Wallis tests. The study found few meaningful differences $(\mathrm{p}<0.05)$ between male and female officials for match characteristics such as total distance covered, time spentengaged in HIR, or in individual effort characteristics such as frequency of HIR or distance covered during each HIR effort. However all significant findings showed only small to medium effect sizes for sex and match time. Findings from the present study suggest that the current guidelinesavailable to international officials should be reevaluated and a removal of sex differences considered.

Key Words: GPS, effort analysis, officiating, exercise intensity, team sports 


\section{INTRODUCTION}

The performance of those officiating (to act as an official [judge]) team sports can strongly influence the outcome of a sporting fixture (Larkin, Berry, Dawson, \& Lay, 2011) which has the potential for wide ranging consequences on sports teams and players alike. The focus on game specific preparation of athletes by conditioning coaches is well established as valuable for improving skill and fitness (Gabbett, Jenkins, \& Abernethy, 2009) but limited literature exists on the demands on match officials. In order for officials to prepare adequately for the demands of the sport they are operating in the in game physical demands need to be ascertained, and while data exists from a number of other sports (Elsworthy and Dascombe, 2011; 0'Hara et al., 2013; Rebelo et al., 2011), the unique officiating scenario in hockey make the transfer of knowledge difficult. Previous research using a sample of male hockey officials $(n=10)$ (Sunderland, Taylor, Pearce, \& Spice, 2011) found that they covered on average $6.66, \mathrm{~s}=0.41 \mathrm{~km}$ per match on average. However, since the publication of this study in 2010 there have been a number of rule amendments to the game which likely change physical demands placed on officials. The primary rule change being the move from two 35 minute halves to four 15 minute quarters. Previous data (Sunderland Taylor, Pearce, \& Spice, 2011) has been collected under the older rule structure, this may result in the conditioning advice that is given to hockey officials being inappropriate for the purposes of preparing them for the modern game. This may be problematic, for example if an official does not have an adequate level of fitness required to officiate a match then it is reasonable to assume he/she may not be able to place themselves in the correct position on the pitch to make accurate decisions (Mallo, Frutos, Juárez, \& Navarro, 2012).

Previous research has focused on male hockey officials and there is a lack of knowledge regarding the demands of officiating female hockey. The current International Hockey Federation (FIH) fitness test requirements for international panel appointment assume a decrease in demand of officiating female hockey compared to the male game (von Reth, 2005). This assumption is supported by research carried out on demands of officiating female and male soccer $(10.03, \mathrm{~s}=0.30$ vs. $11.59, \mathrm{~s}=0.94 \mathrm{~km}$ total distance covered) (Mallo, Veiga, De Subijana, \& Navarro, 2010; Mallo, Navarro, García-Aranda, Gilis, \& Helsen, 2007). As previous studies have demonstrated that there are smallvariations from game to game in total distance measures (Weston, Drust, Atkinson, \& Gregson, 2011) this would infer that there is a sex difference in terms of the total distance covered by officials in their respective matches.

Total distance covered is one metric of interest when investigating match demands, the constituent parts of that total distance are also of importance. Sunderland et al. demonstrated that male hockey officials $(n=10)$ covered on average approximately $9.3 \%$ of the total match distance engaged in high intensity running $\left(\mathrm{HIR},>4.31 \mathrm{~m} \cdot \mathrm{s}^{-1}\right)$ and while there is a large coefficient of variation (CoV; 22.9\%) in the amount of HIR across repeat matches over a short period of time (8 weeks) (Weston, Drust, Atkinson, \& Gregson, 2011) and a strong interrelation 
between players and officials HIR (Weston, Drust, \& Gregson, 2011), understanding these high intensity efforts is vital knowledge for conditioning coaches to assist officials in preparing for the game.

The aim of the present study is to investigate the physical demands of officiating international hockey for both male and females under the new International Hockey Federation (FIH) rules. A further aim is to investigate the constituent parts of officiating performance. 


\section{METHODS}

A cross-sectional observationalstudy was carried out. Match data were collected at one international tournament. Data were collected from 33 male and 33 female matches, with officials taking charge of matches of their own sex. All matches were played over $4 \mathrm{x} 15 \mathrm{~min}$ quarters (Q1-4) according to FIH rules between 10:00 and 20:30 CET with environmental conditions; temperature $25.1, \mathrm{~s}=4.9^{\circ} \mathrm{C}$, pressure $764.31, \mathrm{~s}=2.25 \mathrm{mmHg}$, humidity $41, \mathrm{~s}=10 \%$.

\section{Subjects}

An Institutional Review Board granted ethical approval, and all participants prior to data collection signed an informed consent form. A total of 10 male (mean age; 38.7, s=5.4 years, mean stature; $1.77, \mathrm{~s}=0.07 \mathrm{~m}$, mean mass; $78.4, \mathrm{~s}=8.9 \mathrm{~kg}$ ) having officiated a mean of $91, \mathrm{~s}=54$ international matches (Range $=20-190$ ) and 11 female officials (mean age, 33.5, s=6.5 years; stature; $1.66, \mathrm{~s}=0.04 \mathrm{~m}$, mass; $60.1, \mathrm{~s}=6.6 \mathrm{~kg}$ ) having officiated a mean of $73, \mathrm{~s}=38$ international matches (Range $=39-160$ ), from 13 different nations volunteered and agreed to participate.

\section{Procedures}

All officials were asked to wear a Catapult GPS device (Minimax S4 10hz, Catapult Innovations, Australia) in a bespoke Lycra vest, which ensured the placement of the unit between the scapulae. All devices were fitted to participants by the same researcher one hour prior to the game to allow for them to undertake their usual pre-gameroutines.

All devices were synchronized to the same laptop (Dell Latitude E5520, Dell Technologies, USA) internal clock, and in order to ascertain the exact start and end point of each period of play a synchronization signal was generated on a third device by vigorously shaking it when the whistle sounded. This was repeated to signify the start and end of each match period and these times were applied across the 2 test devices.

The data was then analysed according to the following HIR threshold in line with previous literature (Sunderland et al., 2011) $\left(>4.31 \mathrm{~m} \cdot \mathrm{s}^{-1}\right)$. The frequency, distance covered and duration of each individual movement, or effort, in the HIR zone was calculated using the default manufacturer settings which determined an effort based on the movement meeting either of 2 rules; either the person must spend longer than the set time (" $t$ ") in the zone, e.g.; spend $>1$ second travelling $>4.31 \mathrm{~m} \cdot \mathrm{s}^{-1}$ or a movement must be longer than " $\mathrm{t}$ " with a peak within the zone. An effort is considered finished when the velocity drops by 2 zones.

Catapult Sprint software (v5.14, Catapult Innovations, Australia), was used to download the data and determine the raw data for; match time, total distance covered, $\%$ distance and time spent engaged in HIR and the mean distanceand time of each HIR effort, for both the whole match and individual quarters. The frequency, distance and duration of efforts in HIR as a match mean was also established. This data was then exported into Excel (Microsoft Windows, USA) to 
obtain measures of central tendency.

The total number of data files included in this study was $n=60$ for males, and $n=61$ for females out of a maximum $n=66$ for each sex, this being due to 5 occasions where officials requested not to wear the devices, and 4 due to injuries in the game causing a long stoppage ( $>5 \mathrm{~min}$ ). This resulted in a mean $6, \mathrm{~s}=1$ matches being recorded for each official.

\section{Statistical Analysis}

Statistical Analyses were carried out using IBM SPSS Statistics (v22, IBM SPSS Inc., USA). A Shapiro-Wilk test was used to test for normality in the data with a significance of $p<0.05$ indicating normal distribution, and a Levenes Test was used to test for homogeneity of variance with a significance level of $\mathrm{p}<0.05$ and indicated that the data failed the assumptions required for parametric statistical analysis, therefore a non-parametric Kruskal-Wallis with pair-wise comparisons post-hoc test was used to analyse within-sex differences for match period, and a Man-Whitney U test was used to analyse differences between sexesat all time points. The alpha level was set at $\mathrm{p} \leq 0.05$ and all data presented as means, $\mathrm{s}=$ standard deviations (SD). Effect sizes were calculated using the equation; $=Z / \sqrt{\mathrm{N}}$, with the effect sizes small $(>0.20)$, medium $(>0.50)$ or large $(>0.80)$ (Cohen, 1988).

\section{Results}

\section{Match Characteristics}

There was a non-significant difference for match time between males and females (72.92, $\mathrm{s}=3.86 \mathrm{vs} .72 .45, \mathrm{~s}=4.15 \mathrm{~min})$, however the males had a significantly longer $\mathrm{Q} 4(19.63, \mathrm{~s}=2.80$ $\mathrm{min})$ than the females $(18.35, \mathrm{~s}=2.05)(\mathrm{p}=0.02 r=0.21)$ and the males $\mathrm{Q} 4$ was also significantly longer than their Q1 $(\mathrm{p}<0.001, r=0.46), \mathrm{Q} 2(\mathrm{p}<0.001, r=0.32)$ and Q3 ( $\mathrm{p}=$ $0.01, r=0.29)$, additionally Q3 was significantly longer than Q1 ( $\mathrm{p}=0.03 r=0.42)$, as shown in Table 1. The maximum speed of male officials was significantly greater than that of the females for the match as a whole $(\mathrm{p}<0.001, r=0.34)$, but also in each individual period $(\mathrm{Q} 1, \mathrm{p}<0.001$ $r=0.24 ; \mathrm{Q} 2, \mathrm{p}=0.01, r=0.26 ; \mathrm{Q} 3, \mathrm{p}<0.001, r=0.28 ; \mathrm{Q} 4, \mathrm{p}=0.01, r=0.23$ )

\section{INSERT TABLE 1 HERE}

\section{Official Work Rates}

Table 1 shows the work rate metrics for the male and female officials, both for the whole match and the individual quarters. Females covered distance at a significantly greater rate during Q1 compared to males $(p=0.01 r=0.22$ ) but no other differences were found for either rate of distance covered, or total distance. Males covered a significantly higher percentage of total distance $(p=0.02 r=0.22)$, and spent a significantly greater percentage of time engaged in HIR $(\mathrm{p}=0.04 r=0.19)$ during $\mathrm{Q} 3$ than females, but no further differences occurred for individual 
Q's or for the match as a whole.

\section{Effort Frequency, Distance and Time}

During the whole match, male officials had an average HIR effort distance of $13.70, \mathrm{~s}=2.66 \mathrm{~m}$, duration of $2.78, \mathrm{~s}=0.51 \mathrm{~s}$, and frequency of $37.1, \mathrm{~s}=15.6$, compared to females' distance of 13.29 , $\mathrm{s}=3.00 \mathrm{~m}$, duration of $2.76, \mathrm{~s}=0.59 \mathrm{~s}$ and frequency of $32.3, \mathrm{~s}=16.3$. No significant differences were found between males and females over the whole match. Figure 1 presents the collated HIR effort data across the four quarters. HIR effort duration for female officials was significantly longer for female officials in Q4 compared to Q2 ( $p=0.04 r=0.25)$ and Q3 ( $p=0.03 r=0.26)$. There were no differences between sex for HIR effort duration or effort length during any individual time period, or the whole match. While no statistical differences occurred as a match mean of HIR effort frequency or at any other individual time period, males demonstrated significantly more HIR efforts in Q3 compared to females ( $\mathrm{p}=0.03 r=0.20)$.

\section{INSERT FIGURE 1 HERE}

\section{Discussion}

The aim of the present study was to investigate the demands of officiating international level hockey matches under the currentrules determined by the FIH, and to ascertain differences between officiating male and female hockey in order to inform the practice of conditioning coaches in preparing officials to undertake officiating elite hockey.

A key finding from this study was that there is minimal difference $(r=0.105)$ between males and females in relation to total match distances covered. The current guidelines for officiating international hockey, published in the FIH Umpires Handbook (von Reth, 2005), state lower fitness test requirements for females. However, findings from the present study suggest this should not be the case. Findings also suggest that the rule changes that have occurred in hockey may have resulted in a reduction in intensity. The present study found a rate of distance covered of $80.93, \mathrm{~s}=10.80 \mathrm{~m} \cdot \mathrm{min}^{-1}$ for males and $82.68, \mathrm{~s}=9.35 \mathrm{~m} \cdot \mathrm{min}^{-1}$ for females in comparison to $86.95, \mathrm{~s}=5.35 \mathrm{~m} \cdot \mathrm{min}^{-1}$ found in previous literature (Sunderland et al., 2011). This suggests that current guidelines for fitness training in terms of the total distance requirement are correct as they should prepare officials to undertake relative intensities representative of those experienced duringmatches. However, present findings suggest that there is no difference between males and females and so conditioning coaches should design programs for both to represent an intensity of approximately $82 \mathrm{~m} \cdot \mathrm{min}^{-1}$.

When investigating HIR throughout the whole match, there were no significant differences for sex, there was a trend for the male officials to cover more ground and consequently spend more time engaged in HIR. However, this studydemonstrates that the amount of distance covered in 
HIR for male hockey officials is similar to that found in the previous literature $(9.6, \mathrm{~s}=3.6 \mathrm{vs} .9 .3$, $s=3.2 \%$ ) (Sunderland et al., 2011). The variability that exists in the amount of HIR covered by officials during a match is a possible reason for the lack of significance between sexes, male officials have shown $38.8 \% \mathrm{CoV}$ and females $44.1 \%$. This large variability between matches for HIR has also been reported among soccer officials, with a reported $22.9 \% \mathrm{CoV}$ seen over an 8 week period (Weston et al., 2011). For an exercise practitioner this provid es a baseline for the requirements when designing a training program for officials in the sense that it should contain approximately $9 \%$ of the distance covered in HIR. However, the CoV should suggest caution with using this value as the absolute amount, and training programs should be designed to ensure officials can cope with the upper limit of the demands of matches, approximately $13.5 \%$ of distance and $3.8 \%$ of time. While beyond the scope of this study, it would be of interest to examine the effect of the players HIR on the officials HIR to see if the relationship is the same as demonstrated in soccer (Weston, Drust, \& Gregson, 2011).

The only significant difference between sexes was found in the maximum velocity achieved in each match. Males had significantly greater $(\mathrm{p}<0.001 r=0.34)$ maximum velocity $(5.95, \mathrm{~s}=0.44$ vs. $5.62, \mathrm{~s}=0.44 \mathrm{~m} \cdot \mathrm{s}^{-1}$ ). However, it is not possible to determine if this is a result of the maximum sprint velocity of the males being higher than the females and therefore them having the greater capacity to achieve those speeds, or whether it is as a result of the demands of the matches they are officiating and having to move faster in order to maintain an effective distance from the play.

Some significant differences were found in the data when dividing the game into its constituent quarters of play such as males engaging in significantly greater percentage distance in HIR in Q3 compared to females (9.20, $\mathrm{s}=4.32$ vs. $7.41, \mathrm{~s}=5.19 \%)$, and males covering the distance at a significantly lower rate than females in Q1 (83.78, s=12.95 vs. 86.57, s=14.39) when total distance was normalized to match time, although, no difference was found in either the quarter time, or total distance. Again, while the data showed significant differences, the effect sizes ranged from small to medium, suggesting more than simply the sex of the official influenced the findings such as the tactics of the teams, who may prioritise certain tactics at differing points of the game depending on their sex, and as mentioned previously, this may affect the movement requirements of the officials (Weston, Drust, \& Gregson, 2011).

Previous studies which have investigated matches with a 4-quarter format have demonstrated a decrease in the TD across match periods (Larkin et al., 2014), however this was not demonstrated in the presentstudy. The lack of any effect of match period on TD or relative rate of distance covered suggests that when developing fitness test programs, exercise practitioners need to ensure that officials are capable of maintaining the same level of effort throughout the whole match time.

Also presented in this study is an investigation into the variation in frequency, duration and distance covered of efforts in each movement category, and in doing so this study presents a unique data set with implications for the development of hockey officials training programs. As previously mentioned, while there are significant differences between maleand female officials, the effect sizes associated with these differences range from small to medium suggesting that while there is a difference, this cannot be attributed to differences of sex. However, the findings are of interest to those trying to develop training programs and shows that the mean distance covered in each HIR effort is $13.29 \mathrm{~m}$ for females and $13.70 \mathrm{~m}$ for males, and approximately 35 HIR efforts a game. However, the HIR effort length can vary by around $3 \mathrm{~m}$, and officials can undertake up to 50 HIR efforts a game, so this higher requirement should be considered by 
coaches when devising training programs. Furthermore, female officials need to be prepared for longer distance efforts in the final quarter compared to their earlier quarters, and while it is not possible to determine from this study if this is due to a decision being made by the officials or as a result of the demands of the play it should still be considered by conditioning coaches.

An unexpected consequence of the addition of video referrals to the international hockey game, and their current utilization tactics by the teams is the significantly longer $4^{\text {th }}$ quarter when compared to other match periods for males, something which is not demonstrated in the female game despite the universal application of the rule. While it is acknowledged this is beyond the control of the officials and there is no significant change in work rate demands across quarters meaning that there is no additional training required manage the additional match time, it is worth the officials keeping this in mind.

The present study has demonstrated an updated view of the demands of officiating elite hockey, and suggests that there has been a decrease in the requirement for total distance covered between the present and previous research. It also shows that while there are differences in the data, the lack of effect sizes suggests that there is sufficient match to match variability and overlap in the requirements of officiating female and male hockey that they should be considered as one group.

In terms of the FIH guidelines, the present study suggests they should consider treating male and female officials as one group, as the data presented in this study does not show sufficient effect sizes to separate the groups in terms of distance covered or the amount of HIR. Therefore, coaches should be looking to ensure their officials are capable of comfortably completing approximately $83 \mathrm{~m}$ per minute of match time, with $9.5 \%$ of the distance covered at speeds $>4.31 \mathrm{~m} \cdot \mathrm{s}^{-1}$, but caution should always be used with these mean figuresas the CoVin the amount of HIR carried out suggests officials could be required to cover up to $13.5 \%$ of the match distance while engaged in HIR, and conditioning coaches should prepare them to be able to do so.

Finally, where attention should be paid is in the development of training programs which should look to utilize the individual effort characteristics presented in this study in order to better prepare officials for match performances. Where possible coaches should design training efforts around a mean HIR effort length of approximately $13.5 \mathrm{~m}$, and for them to carry out 35 per match, although they should be prepared to undertake efforts up to $3 \mathrm{~m}$ longer or shorter, and up to 50 per match. 
Cohen, J. (1988). Statistical power analysis for the behavioural sciences. hillside. NJ: Lawrence Earlbaum Associates,

Elsworthy, N., \& Das combe, B. J. (2011). The match demands of Australian rules football umpires in a statebased competition. International Journal of Sports Physiologyand Performance, 6(4), 559-571.

Gabbett, T., Jenkins, D., \& Abernethy, B. (2009). Game-based training for improving skill and physical fitness in teamsport athletes. International Journal of Sports Science \& Coaching, 4(2), 273-283.

Larkin, P., Berry, J., Dawson, B., \& Lay, B. (2011). Perceptual and decision-making skills of australian football umpires. International Journal of Performance Analysis in Sport, 11(3), 427-437.

Larkin, P., O’Brien, B., Mesagno, C., Berry, J., Harvey, J., \& Spittle, M. (2014). Assessment of decisionmaking performance and in-game physical exertion of australian football umpires. Journal ofSports Sciences, 32(15), 1446-1453.

Mallo, J., Veiga, S., De Subijana, C. L., \& Navarro, E. (2010). Activity profile of top-class female soccer refereeing in relation to the position of the ball. Journal ofScience and Medicine in Sport, 13(1), 129-132.

Mallo, J., Frutos, P. G., Juárez, D., \& Navarro, E. (2012). Effect of positioning on the accuracy of decision making of ass ociation football top-class referees and as sistant referees during competitive matches. Journal of Sports Sciences, 30(13), 1437-1445.

Mallo, J., Navarro, E., García-Aranda, J., Gilis, B., \& Helsen, W. (2007). Activity profile of top-class ass ociation football referees in relation to performance in selected physical tests. JournalofSports Sciences, 25(7), 805-813.

O'Hara, J. P., Brightmore, A., Till, K., Mitchell, I., Cummings, S., \& Cooke, C. B. (2013). Evaluation of movement and physiological demands of rugby league referees using global positioning systems tracking. Internal Journal of Sports Medicine, 34(9), 825-831.

Rebelo, A. N., Ascensão, A. A., Magalhães, J. F., Bishoff, R., Bendiksen, M., \& Krustrup, P. (2011). Elite futsal refereeing: Activity profile and physiological demands. Journal of Strength \& Conditioning Research, 25(4), 980-987

Sunderland, C., Taylor, E., Pearce, E., \& Spice, C. (2011). Activity profile and physical demands of male field hockey umpires in international matches. European Journal of Sport Science, 11(6), 411-417.

von Reth, P. (2005). FIH international umpires handbook (1st ed.) International Hockey Federation.

Weston, M., Drust, B., G., \& Gregson, W. (2011). Intensities of exercis e during match-play in FA Premier League referees and players. Journalof Sports Sciences, 29(05), 527-532.

Weston, M., Drust, B., Atkinson, G., \& Gregson, W.(2011). Variability of soccer referees’ match performances. International JournalofSports Medicine, 32(03), 190-194. 